\title{
TRANSIENT SOLUTION \\ TO THE TIME-DEPENDENT MULTISERVER POISSON QUEUE
}

\author{
B. H. MARGOLIUS, ${ }^{*}$ Cleveland State University
}

\begin{abstract}
We derive an integral equation for the transient probabilities and expected number in the queue for the multiserver queue with Poisson arrivals, exponential service for timevarying arrival and departure rates, and a time-varying number of servers. The method is a straightforward application of generating functions. We can express $p_{\hat{c}-1}(t)$, the probability that $\hat{c}-1$ customers are in the queue or being served, in terms of a Volterra equation of the second kind, where $\hat{c}$ is the maximum number of servers working during the day. Each of the other transient probabilities is expressed in terms of integral equations in $p_{\hat{c}-1}(t)$ and the transition probabilities of a certain time-dependent random walk. In this random walk, the rate of steps to the right equals the arrival rate of the queue and the rate of steps to the left equals the departure rate of the queue when all servers are busy.

Keywords: Transient probability; time-dependent Poisson arrival rate; time-dependent exponential service rate; time-dependent queue; multiserver queue; single-server queue; modified Bessel function
\end{abstract}

2000 Mathematics Subject Classification: Primary 60K25

Secondary $60 \mathrm{~J} 35$

\section{Introduction}

Many queueing systems have time-varying arrival and departure rates. In this paper, we are motivated by police calls for service, which occur more frequently in the evening - when the bars close - and during morning rush hour than in the dead of night, when residents are asleep (see Figure 1). Relatively few exact results exist for transient transition probabilities because computations tend to be fairly involved even in the case of constant rates. Here, we present a method of computing the transition probability $p_{i, n}(s, t)$, the probability that there are $n$ customers in the queue at time $t$ given that there were $i$ customers in the queue at time $s$, for a multiserver queue with time-varying arrival and departure rates. We also derive an integral equation for the expected number in the system. In the case of constant parameters, these formulae are consistent with those found by Parthasarathy and Sharafali [3].

The paper is organized as follows. In Section 2, we derive an integral equation for the transient probability distribution for the $\mathrm{M}_{t} / \mathrm{M}_{t} / c$ queue and find a formula for the expected number in the queue at time $t$. The transient probabilities and the expected number in the queue are expressed in terms of integral equations in $p_{c-1}(t)$, the transient probability that there are $c-1$ customers in the system. We write $p_{c-1}(t)$ in terms of a Volterra equation of the second kind. In Section 3, we extend the results of Section 2 to the case of a time-varying

Received 22 June 2004; revision received 16 December 2004.

* Postal address: Department of Mathematics, 1515 Rhodes Tower, 2121 Euclid Avenue, Cleveland, OH 44114-2214, USA. Email address: b.margolius@csuohio.edu 

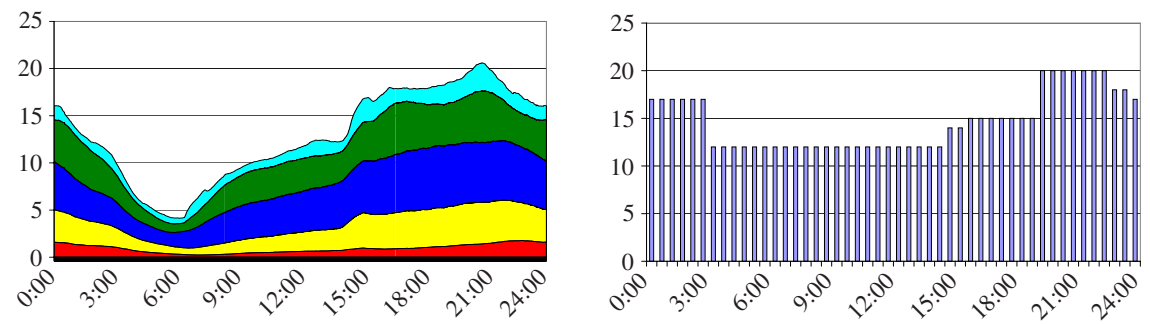

Figure 1: Data from the 4th District of the City of Cleveland. The left-hand panel shows the hourly rate of police calls for service versus time of day. The bands indicate, in increasing order from top to bottom, the priority of calls. The right-hand panel shows the staffing plan for police cars versus time of day.

number of servers. In Section 4, we provide a condition for asymptotic stability in the case of a time-varying number of servers and, in Section 5, we provide numerical examples.

\section{The transient probability distribution for the $M_{t} / \mathbf{M}_{t} / c$ queue}

We can write the Chapman-Kolmogorov forward differential equations for a time-dependent multiserver queue with $c$ servers as

$$
\begin{aligned}
\dot{p}_{i, 0}(s, t)= & -p_{i, 0}(s, t) \lambda(t)+p_{i, 1}(s, t) \mu(t), \\
\dot{p}_{i, n}(s, t)= & -p_{i, n}(s, t)(\lambda(t)+n \mu(t))+p_{i, n-1}(s, t) \lambda(t) \\
& +(n+1) p_{i, n+1}(s, t) \mu(t), \quad 1 \leq n<c, \\
\dot{p}_{i, n}(s, t)= & -p_{i, n}(s, t)(\lambda(t)+c \mu(t))+p_{i, n-1}(s, t) \lambda(t) \\
& +c p_{i, n+1}(s, t) \mu(t), \quad c \leq n,
\end{aligned}
$$

and

$$
p_{i, n}(s, s+)=\delta(n, i):= \begin{cases}1 & \text { if } n=i, \\ 0 & \text { otherwise, }\end{cases}
$$

where, recall, $p_{i, n}(s, t)$ is the probability that there are $n$ customers in the system at time $t$ given that there were $i$ customers at time $s, \lambda(t)>0$ is the time-varying arrival rate of the queue, $\mu(t)>0$ is the service rate, and a dot denotes a time derivative. We will usually write $p_{n}(t)$ for $p_{i, n}(s, t)$. We define the generating function $P_{c}(z, t)$ for the number of customers awaiting commencement of service (again suppressing the dependence on $s$ and $i$ in the notation) as

$$
P_{c}(z, t)=\sum_{n=0}^{c-1} p_{n}(t)+\sum_{n=c}^{\infty} z^{n-c+1} p_{n}(t)=q_{c-1}(t)+\sum_{n=1}^{\infty} z^{n} p_{n+c-1}(t),
$$

where $q_{m}(t)=\sum_{n=0}^{m} p_{n}(t)$. Note that

$$
P_{c}(z, s)=1, \quad i<c, \quad \text { and } \quad P_{c}(z, s)=z^{i-c+1}, \quad i \geq c .
$$

Using (2.1)-(2.3), and the definition of the generating function $P_{c}(z, t)$, we obtain the following differential equation:

$$
\begin{aligned}
& \dot{P}_{c}(z, t) \\
& \quad=\left(z \lambda(t)-(\lambda(t)+c \mu(t))+c \mu(t) z^{-1}\right)\left(P_{c}(z, t)-q_{c-1}(t)\right)+\lambda(t) p_{c-1}(t)(z-1) .
\end{aligned}
$$


This has the solution

$$
\begin{aligned}
P_{c}(z, t)= & \int_{s}^{t} \Phi_{c, z}(\eta, t)\left(\lambda(\eta) p_{c-1}(\eta)(z-1)\right. \\
& \left.-\left(z \lambda(\eta)-(\lambda(\eta)+c \mu(\eta))+c \mu(\eta) z^{-1}\right) q_{c-1}(\eta)\right) \mathrm{d} \eta \\
& +\Phi_{c, z}(s, t) P_{c}(z, s) \\
= & \int_{s}^{t} \Phi_{c, z}(\eta, t)\left(c \mu(\eta) p_{c-1}(\eta)\left(1-z^{-1}\right)\right. \\
& \left.-\left(z \lambda(\eta)-(\lambda(\eta)+c \mu(\eta))+c \mu(\eta) z^{-1}\right) q_{c-2}(\eta)\right) \mathrm{d} \eta \\
& +\Phi_{c, z}(s, t) P_{c}(z, s),
\end{aligned}
$$

where

$$
\Phi_{c, z}(s, t)=\exp \left\{\int_{s}^{t}\left(z \lambda(\eta)-(\lambda(\eta)+c \mu(\eta))+c \mu(\eta) z^{-1}\right) \mathrm{d} \eta\right\} .
$$

Using integration by parts and the facts that

$$
\begin{aligned}
\dot{q}_{c-2}(t) & =-\lambda(t) p_{c-2}(t)+(c-1) \mu(t) p_{c-1}(t) \\
\frac{\partial}{\partial s} \Phi_{c, z}(s, t) & =-\left(z \lambda(s)-(\lambda(s)+c \mu(s))+c \mu(s) z^{-1}\right) \Phi_{c, z}(s, t),
\end{aligned}
$$

we obtain

$$
\begin{aligned}
P_{c}(z, t)= & \int_{s}^{t} \Phi_{c, z}(\eta, t)\left(c \mu(\eta) p_{c-1}(\eta)\left(1-z^{-1}\right)\right. \\
& \left.-\left(-\lambda(\eta) p_{c-2}(\eta)+(c-1) \mu(\eta) p_{c-1}(\eta)\right)\right) \mathrm{d} \eta \\
& +\left.q_{c-2}(\eta) \Phi_{c, z}(\eta, t)\right|_{\eta=s} ^{\eta=t}+\Phi_{c, z}(s, t) P_{c}(z, s) \\
= & \int_{s}^{t} \Phi_{c, z}(\eta, t)\left(\mu(\eta) p_{c-1}(\eta)\left(1-c z^{-1}\right)+\lambda(\eta) p_{c-2}(\eta)\right) \mathrm{d} \eta \\
& +q_{c-2}(t)-q_{c-2}(s) \Phi_{c, z}(s, t)+\Phi_{c, z}(s, t) P_{c}(z, s) .
\end{aligned}
$$

Define

$$
\tilde{I}_{n}(s, t)= \begin{cases}\sum_{k=0}^{\infty} \frac{\Lambda^{n+k}(s, t)}{(n+k) !} \frac{c^{k} M^{k}(s, t)}{k !} \exp \{-\Lambda(s, t)-c M(s, t)\} & \text { if } n \geq 0, \\ \sum_{k=0}^{\infty} \frac{\Lambda^{k}(s, t)}{k !} \frac{c^{k-n} M^{k-n}(s, t)}{(k-n) !} \exp \{-\Lambda(s, t)-c M(s, t)\} & \text { if } n<0,\end{cases}
$$

where $\Lambda(s, t)=\int_{s}^{t} \lambda(\eta) \mathrm{d} \eta$ and $M(s, t)=\int_{s}^{t} \mu(\eta) \mathrm{d} \eta$. Note that $\tilde{I}_{n}(s, t)$ is the probability of a random walk, with time-dependent rates $\lambda(t)$ for steps to the right and $c \mu(t)$ for steps to the left, making $n$ more steps to the right than it does to the left during the time interval from $s$ to $t$. The probability $\tilde{I}_{n}(s, t)$ is related to the modified Bessel function $I_{n}(x)$. Recall that

$$
I_{n}(x)=\left(\frac{x}{2}\right)^{n} \sum_{k=0}^{\infty} \frac{\left(\frac{1}{2} x\right)^{2 k}}{(n+k) ! k !}
$$


hence, we can write

$$
\tilde{I}_{n}(s, t)=\left(\frac{\Lambda(s, t)}{c M(s, t)}\right)^{n / 2} I_{n}(2 \sqrt{\Lambda(s, t) c M(s, t)}) \exp \{-\Lambda(s, t)-c M(s, t)\}
$$

and $\tilde{I}_{0}(t, t)=I_{0}(0)=1$. The $\tilde{I}_{n}(s, t), n \in \mathbb{N}$, have the property that

$$
\begin{aligned}
\frac{\partial}{\partial t} \tilde{I}_{n}(s, t) & =\lambda(t) \tilde{I}_{n-1}(s, t)-(\lambda(t)+c \mu(t)) \tilde{I}_{n}(s, t)+c \mu(t) \tilde{I}_{n+1}(s, t), \\
\frac{\partial}{\partial s} \tilde{I}_{n}(s, t) & =-\lambda(s) \tilde{I}_{n-1}(s, t)+(\lambda(s)+c \mu(s)) \tilde{I}_{n}(s, t)-c \mu(s) \tilde{I}_{n+1}(s, t) .
\end{aligned}
$$

It can be shown that

$$
\Phi_{c, z}(s, t)=\sum_{n=-\infty}^{\infty} z^{n} \tilde{I}_{n}(s, t)
$$

(see, for example, [4, Appendix B]). Using (2.6) and the fact that the coefficient of $z^{n}$ in (2.5) is $p_{n+c-1}(t)$, we can write a formula for the probability that there are $n+c-1$ customers in the queue for $n>0$ :

$$
\begin{aligned}
p_{n+c-1}(t)= & \int_{s}^{t}\left(\mu(\eta) p_{c-1}(\eta)\left(\tilde{I}_{n}(\eta, t)-c \tilde{I}_{n+1}(\eta, t)\right)+\lambda(\eta) p_{c-2}(\eta) \tilde{I}_{n}(\eta, t)\right) \mathrm{d} \eta \\
& +\tilde{I}_{n-i+c-1}(s, t)\left(1-q_{c-1}(s)\right)+q_{c-1}(s) \tilde{I}_{n}(s, t) .
\end{aligned}
$$

For $n=0$, we have

$$
\begin{aligned}
q_{c-1}(t)= & \int_{s}^{t}\left(\mu(\eta) p_{c-1}(\eta)\left(\tilde{I}_{0}(\eta, t)-c \tilde{I}_{1}(\eta, t)\right)+\lambda(\eta) p_{c-2}(\eta) \tilde{I}_{0}(\eta, t)\right) \mathrm{d} \eta \\
& +q_{c-2}(t)+\tilde{I}_{-i+c-1}(s, t)\left(1-q_{c-1}(s)\right)+q_{c-1}(s) \tilde{I}_{0}(s, t),
\end{aligned}
$$

meaning that

$$
\begin{aligned}
p_{c-1}(t)= & \int_{s}^{t}\left(\mu(\eta) p_{c-1}(\eta)\left(\tilde{I}_{0}(\eta, t)-c \tilde{I}_{1}(\eta, t)\right)+\lambda(\eta) p_{c-2}(\eta) \tilde{I}_{0}(\eta, t)\right) \mathrm{d} \eta \\
& +\tilde{I}_{-i+c-1}(s, t)\left(1-q_{c-1}(s)\right)+q_{c-1}(s) \tilde{I}_{0}(s, t),
\end{aligned}
$$

since $q_{c-1}(t)-q_{c-2}(t)=p_{c-1}(t)$. From (2.1) and (2.2), using the equation for $\dot{p}_{c-2}(t)$ gives

$$
\dot{\boldsymbol{p}}(t)=\boldsymbol{p}(t) \boldsymbol{A}(t)+(c-1) \mu(t) p_{c-1}(t) \boldsymbol{e}_{c-1},
$$

where

$$
\boldsymbol{A}(t)=\left(\begin{array}{cccccc}
-\lambda(t) & \lambda(t) & 0 & \cdots & \cdots & 0 \\
\mu(t) & -\lambda(t)-\mu(t) & \lambda(t) & & & 0 \\
0 & 2 \mu(t) & -\lambda(t)-2 \mu(t) & \ddots & & 0 \\
\vdots & & \ddots & \ddots & \ddots & \vdots \\
\vdots & & & \ddots & \ddots & \lambda(t) \\
0 & 0 & 0 & \cdots & (c-2) \mu(t) & -\lambda(t)-(c-2) \mu(t)
\end{array}\right),
$$


$\boldsymbol{e}_{c-1}$ is a row vector of length $c-1$ with 0 in the first $c-2$ places and 1 in the last place, and $\boldsymbol{p}(t)$ is the row vector

$$
\boldsymbol{p}(t)=\left(p_{0}(t), p_{1}(t), \ldots, p_{c-2}(t)\right) .
$$

Let $\boldsymbol{U}(s, t)$ be an evolution operator such that $\boldsymbol{U}(t, t)=\boldsymbol{I}$ (the identity operator) and let $(\partial / \partial t) \boldsymbol{U}(s, t)=\boldsymbol{U}(s, t) \boldsymbol{A}(t)$. Then

$$
\boldsymbol{p}(t)=\int_{s}^{t}(c-1) \mu(v) p_{c-1}(v) \boldsymbol{e}_{c-1} \boldsymbol{U}(v, t) \mathrm{d} v+\boldsymbol{p}(s) \boldsymbol{U}(s, t) .
$$

We can solve for $\boldsymbol{U}(s, t)$ numerically using techniques for solving linear systems of ordinary differential equations. For each fixed $s$ and $t, \boldsymbol{U}(s, t)$ is a square matrix whose components we index from 0 to $c-2$. In particular, $\boldsymbol{U}_{\cdot, c-2}(s, t)$ is the $(c-2)$ th (i.e. final) column of this matrix and $u_{c-2, c-2}(s, t)$ is the element in row $c-2$, column $c-2$. Then, $p_{c-2}(t)$ is given by the formula

$$
p_{c-2}(t)=\int_{s}^{t}(c-1) \mu(v) p_{c-1}(v) u_{c-2, c-2}(v, t) \mathrm{d} v+\boldsymbol{p}(s) \boldsymbol{U}_{\cdot, c-2}(s, t) .
$$

With this information, we can write $p_{c-1}(t)$ in terms of a Volterra equation of the second kind:

$$
\begin{aligned}
p_{c-1}(t)= & \int_{s}^{t}\left[\mu(\eta) p_{c-1}(\eta)\left(\tilde{I}_{0}(\eta, t)-c \tilde{I}_{1}(\eta, t)+(c-1) \int_{\eta}^{t} u_{c-2, c-2}(\eta, v) \lambda(\nu) \tilde{I}_{0}(\nu, t) \mathrm{d} v\right)\right. \\
& \left.+\boldsymbol{p}(s) \boldsymbol{U} \cdot, c-2(s, \eta) \lambda(\eta) \tilde{I}_{0}(\eta, t)\right] \mathrm{d} \eta \\
& +p_{c-1}(s) \tilde{I}_{0}(s, t)+\tilde{I}_{-i+c-1}(s, t)\left(1-q_{c-1}(s)\right) .
\end{aligned}
$$

Equations (2.7), (2.8), and (2.9), taken together, yield the complete transient distribution for the time-dependent multiserver queue.

\section{Time-varying numbers of servers}

We are interested in applying this technique to queues with time-varying numbers of servers and time-dependent periodic arrivals. In this section, we take $\mu(t)=\mu$, a constant, but permit $c$ and $\lambda(t)$ to vary. (The analysis can easily be extended to the case in which $\mu$ also varies.) Define $\hat{c}=\max _{t} c(t)$.

The analysis proceeds as before. We can write the Chapman-Kolmogorov forward differential equations for a time-dependent multiserver queue with $c(t)$ servers and constant service rate $\mu$ as

$$
\begin{aligned}
\dot{p}_{i, 0}(s, t)= & -p_{i, 0}(s, t) \lambda(t)+p_{i, 1}(s, t) \mu, \\
\dot{p}_{i, n}(s, t)= & -p_{i, n}(s, t)(\lambda(t)+\min \{n, c(t)\} \mu)+p_{i, n-1}(s, t) \lambda(t) \\
& +p_{i, n+1}(s, t) \min \{(n+1), c(t)\} \mu, \quad 1 \leq n<\hat{c}, \\
\dot{p}_{i, n}(s, t)= & -p_{i, n}(s, t)(\lambda(t)+c(t) \mu)+p_{i, n-1}(s, t) \lambda(t) \\
& +p_{i, n+1}(s, t) c(t) \mu,
\end{aligned}
$$

and

$$
p_{i, n}(s, s+)=\delta(n, i),
$$


where, as before, $p_{i, n}(s, t)$ is the probability that there are $n$ customers in the system at time $t$ given that there were $i$ customers at time $s$. (Again, we will usually write $p_{n}(t)$ for this quantity.) We define the probability generating function $P_{c(t)}(z, t)$ as

$$
P_{c(t)}(z, t)=\sum_{n=0}^{\hat{c}-1} p_{n}(t)+\sum_{n=\hat{c}}^{\infty} z^{n-\hat{c}+1} p_{n}(t)=q_{\hat{c}-1}(t)+\sum_{n=1}^{\infty} z^{n} p_{n+\hat{c}-1}(t) .
$$

Using (3.1)-(3.3), and the definition of the generating function $P_{c(t)}(z, t)$, we obtain the following differential equation:

$$
\begin{aligned}
& \dot{P}_{c(t)}(z, t) \\
& \quad=\left(z \lambda(t)-(\lambda(t)+c(t) \mu)+c(t) \mu z^{-1}\right)\left(P_{c(t)}(z, t)-q_{\hat{c}-1}(t)\right)+\lambda(t) p_{\hat{c}-1}(t)(z-1) .
\end{aligned}
$$

This has the solution

$$
\begin{aligned}
P_{c(t)}(z, t)= & \int_{s}^{t} \Phi_{c(t), z}(\eta, t)\left(\lambda(\eta) p_{\hat{c}-1}(\eta)(z-1)\right. \\
& \left.\quad-\left(z \lambda(\eta)-(\lambda(\eta)+c(\eta) \mu)+c(\eta) \mu z^{-1}\right) q_{\hat{c}-1}(\eta)\right) \mathrm{d} \eta \\
& +\Phi_{c(t), z}(s, t) P_{c(t)}(z, s) \\
= & \int_{s}^{t} \Phi_{c(t), z}(\eta, t)\left(c(\eta) \mu p_{\hat{c}-1}(\eta)\left(1-z^{-1}\right)\right. \\
& +\Phi_{c(t), z}(s, t) P_{c(t)}(z, s) \\
= & \int_{s}^{t} \Phi_{c(t), z}(\eta, t)\left(c(\eta) \mu p_{\hat{c}-1}(\eta)\left(1-z^{-1}\right)\right. \\
& +\left.q_{\hat{c}-2}(\eta) \Phi_{c(t), z}(\eta, t)\right|_{\eta=s} ^{\eta=t}+\Phi_{c(t), z}(s, t) P_{c(t)}(z, s) \\
= & \int_{s}^{t} \Phi_{c(t), z}(\eta, t)\left(\mu p_{\hat{c}-1}(\eta)\left(\max \{0, c(\eta)-\hat{c}+1\}-c(\eta) z^{-1}\right)+\lambda(\eta) p_{\hat{c}-2}(\eta)\right) \mathrm{d} \eta \\
& +q_{\hat{c}-2}(t)-q_{\hat{c}-2}(s) \Phi_{c(t), z}(s, t)+\Phi_{c(t), z}(s, t) P_{c(t)}(z, s),
\end{aligned}
$$

where

$$
\Phi_{c(t), z}(s, t)=\exp \left\{\int_{s}^{t}\left(z \lambda(\eta)-(\lambda(\eta)+c(\eta) \mu)+c(\eta) \mu z^{-1}\right) \mathrm{d} \eta\right\} .
$$

Using this, we can write a formula for the probability that there are $n+\hat{c}-1$ customers in the queue for $n>0$ :

$$
\begin{aligned}
& p_{n+\hat{c}-1}(t) \\
& =\int_{s}^{t}\left(\mu p_{\hat{c}-1}(\eta)\left(\max \{0, c(\eta)-\hat{c}+1\} \tilde{I}_{n}(\eta, t)-c(\eta) \tilde{I}_{n+1}(\eta, t)\right)+\lambda(\eta) p_{\hat{c}-2}(\eta) \tilde{I}_{n}(\eta, t)\right) \mathrm{d} \eta \\
& \quad+\tilde{I}_{n-i+\hat{c}-1}(s, t)\left(1-q_{c-1}(s)\right)+q_{\hat{c}-1}(s) \tilde{I}_{n}(s, t) .
\end{aligned}
$$


For $n=0$, we have

$$
\begin{aligned}
& q_{\hat{c}-1}(t) \\
& =\int_{s}^{t}\left(\mu p_{\hat{c}-1}(\eta)\left(\max \{0, c(\eta)-\hat{c}+1\} \tilde{I}_{0}(\eta, t)-c(\eta) \tilde{I}_{1}(\eta, t)\right)+\lambda(\eta) p_{\hat{c}-2}(\eta) \tilde{I}_{0}(\eta, t)\right) \mathrm{d} \eta \\
& \quad+q_{\hat{c}-2}(t)+\tilde{I}_{-i+\hat{c}-1}(s, t)\left(1-q_{c-1}(s)\right)+q_{\hat{c}-1}(s) \tilde{I}_{0}(s, t),
\end{aligned}
$$

meaning that

$$
\begin{aligned}
& p_{\hat{c}-1}(t) \\
& =\int_{s}^{t}\left(\mu p_{\hat{c}-1}(\eta)\left(\max \{0, c(\eta)-\hat{c}+1\} \tilde{I}_{0}(\eta, t)-c(\eta) \tilde{I}_{1}(\eta, t)\right)+\lambda(\eta) p_{\hat{c}-2}(\eta) \tilde{I}_{0}(\eta, t)\right) \mathrm{d} \eta \\
& \quad+\tilde{I}_{-i+\hat{c}-1}(s, t)\left(1-q_{\hat{c}-1}(s)\right)+q_{\hat{c}-1}(s) \tilde{I}_{0}(s, t) .
\end{aligned}
$$

Now we write

$$
\dot{\boldsymbol{p}}(t)=\boldsymbol{p}(t) \boldsymbol{A}(t)+\min \{c(t), \hat{c}-1\} \mu p_{\hat{c}-1}(t) \boldsymbol{e}_{\hat{c}-1},
$$

which has the solution

$$
\boldsymbol{p}(t)=\int_{s}^{t} \min \{c(v), \hat{c}-1\} \mu p_{\hat{c}-1}(v) \boldsymbol{e}_{\hat{c}-1} \boldsymbol{U}(v, t) \mathrm{d} v+\boldsymbol{p}(s) \boldsymbol{U}(s, t),
$$

where $\boldsymbol{U}(s, t)$ is defined as above, with the coefficient of $\mu(t)$ now replaced by $\min \{c(t), j\}$ in row $j$ of the matrix $\boldsymbol{A}(t)$. In particular, $p_{\hat{c}-2}(t)$ is given by

$$
p_{\hat{c}-2}(t)=\int_{s}^{t} u_{c-2, c-2}(v, t) \min \{c(v), \hat{c}-1\} \mu p_{\hat{c}-1}(v) \mathrm{d} v+\boldsymbol{p}(s) \boldsymbol{U}_{\cdot, c-2}(s, t) .
$$

With this information, we can write $p_{\hat{c}-1}(t)$ in terms of a Volterra equation of the second kind:

$$
\begin{aligned}
p_{\hat{c}-1}(t)=\int_{s}^{t}\left[\mu p _ { \hat { c } - 1 } ( \eta ) \left(\max \{0, c(\eta)-\hat{c}+1\} \tilde{I}_{0}(\eta, t)-c(\eta) \tilde{I}_{1}(\eta, t)\right.\right. \\
\left.\quad+\min \{c(\eta), \hat{c}-1\} \int_{\eta}^{t} u_{c-2, c-2}(\eta, v) \lambda(v) \tilde{I}_{0}(\nu, t) \mathrm{d} v\right) \\
\left.\quad+\boldsymbol{p}(s) \boldsymbol{U}_{\cdot, c-2}(s, \eta) \lambda(\eta) \tilde{I}_{0}(\eta, t)\right] \mathrm{d} \eta \\
+q_{\hat{c}-1}(s) \tilde{I}_{0}(s, t)+\tilde{I}_{-i+\hat{c}-1}(s, t)\left(1-q_{\hat{c}-1}(s)\right),
\end{aligned}
$$

where $\boldsymbol{U}_{\cdot, c-2}(s, \eta)$ and $u_{c-2, c-2}(\eta, v)$ are defined as above. If $i<\hat{c}-1$, this simplifies to

$$
\begin{gathered}
p_{\hat{c}-1}(t)=\int_{s}^{t}\left[\mu p _ { \hat { c } - 1 } ( \eta ) \left(\max \{0, c(\eta)-\hat{c}+1\} \tilde{I}_{0}(\eta, t)-c(\eta) \tilde{I}_{1}(\eta, t)\right.\right. \\
\left.\quad+\min \{c(\eta), \hat{c}-1\} \int_{\eta}^{t} u_{c-2, c-2}(\eta, v) \lambda(v) \tilde{I}_{0}(\nu, t) \mathrm{d} v\right) \\
\left.+u_{i, c-2}(s, \eta) \lambda(\eta) \tilde{I}_{0}(\eta, t)\right] \mathrm{d} \eta+\tilde{I}_{0}(s, t) .
\end{gathered}
$$


If $i \geq \hat{c}-1$, we have

$$
\begin{aligned}
p_{\hat{c}-1}(t)= & \int_{s}^{t}\left[\mu p _ { \hat { c } - 1 } ( \eta ) \left(\max \{0, c(\eta)-\hat{c}+1\} \tilde{I}_{0}(\eta, t)-c(\eta) \tilde{I}_{1}(\eta, t)\right.\right. \\
& \left.\left.\quad+\min \{c(\eta), \hat{c}-1\} \int_{\eta}^{t} u_{c-2, c-2}(\eta, v) \lambda(v) \tilde{I}_{0}(v, t) \mathrm{d} v\right)\right] \mathrm{d} \eta \\
& +\tilde{I}_{-i+\hat{c}-1}(s, t)
\end{aligned}
$$

and, in particular, for $i=\hat{c}-1$ we have

$$
\begin{aligned}
p_{\hat{c}-1}(t)= & \int_{s}^{t}\left[\mu p _ { \hat { c } - 1 } ( \eta ) \left(\max \{0, c(\eta)-\hat{c}+1\} \tilde{I}_{0}(\eta, t)-c(\eta) \tilde{I}_{1}(\eta, t)\right.\right. \\
& \left.\left.\quad+\min \{c(\eta), \hat{c}-1\} \int_{\eta}^{t} u_{c-2, c-2}(\eta, v) \lambda(v) \tilde{I}_{0}(\nu, t) \mathrm{d} v\right)\right] \mathrm{d} \eta \\
& +\tilde{I}_{0}(s, t) .
\end{aligned}
$$

Let $X(t)$ be the counting process that gives the number in the queue at time $t$, and define $\mathrm{E}[X(t)]$ as the expected number in the queue at time $t$. Then $\mathrm{E}[X(t)]$ is given by the formula

$$
\mathrm{E}[X(t)]=\mathrm{E}[X(s)]+\Lambda(s, t)-\mu \int_{s}^{t} c(v) \mathrm{d} v+\mu \int_{s}^{t} \sum_{j=0}^{\hat{c}-1} \max \{c(v)-j, 0\} p_{j}(v) \mathrm{d} v .
$$

By assumption $N(s)=i$, and substituting for $p_{j}(v)$ yields

$$
\begin{aligned}
\mathrm{E}[X(t)]= & i+\Lambda(s, t)-\mu \int_{s}^{t} c(v) \mathrm{d} v \\
+ & +\mu \int_{s}^{t} \sum_{j=0}^{t} \max \{c(v)-j, 0\} \\
& \times\left[\int_{s}^{v} u_{\hat{c}-2, j}(\eta, v) \min \{c(\eta), \hat{c}-1\} \mu p_{\hat{c}-1}(\eta) \mathrm{d} \eta+u_{i, j}(s, v)\right] \mathrm{d} v
\end{aligned}
$$

when $i<\hat{c}-1$, and

$$
\begin{aligned}
\mathrm{E}[X(t)]= & i+\Lambda(s, t)-\mu \int_{s}^{t} c(v) \mathrm{d} v \\
& +\mu \int_{s}^{t} \sum_{j=0}^{\hat{c}-1} \max \{c(v)-j, 0\}\left[\int_{s}^{v} u_{\hat{c}-2, j}(\eta, v) \min \{c(\eta), \hat{c}-1\} \mu p_{\hat{c}-1}(\eta) \mathrm{d} \eta\right] \mathrm{d} v
\end{aligned}
$$

when $i \geq \hat{c}-1$. Changing the order of integration and summing before integrating yields

$$
\begin{aligned}
\mathrm{E}[X(t)]= & i+\Lambda(s, t)-\mu \int_{s}^{t} c(v) \mathrm{d} v \\
& +\mu^{2} \int_{s}^{t}\left[p_{\hat{c}-1}(\eta) \min \{c(\eta), \hat{c}-1\} \int_{\eta}^{t} \sum_{j=0}^{\hat{c}-1} \max \{c(v)-j, 0\} u_{\hat{c}-2, j}(\eta, v) \mathrm{d} v\right] \mathrm{d} \eta .
\end{aligned}
$$




\section{Stability}

The queue length process $X(t)$ for the periodic $\mathrm{M}_{t} / \mathrm{M}_{t} / c_{t}$ queue does not converge in distribution as $t \rightarrow \infty$. We therefore consider the concepts of asymptotic stability for stochastic processes that do not converge in distribution [2].

For each $t \geq 0$, let $F_{t}$ be the cumulative distribution function (CDF) of $X(t)$. Let $\mathcal{L} \equiv \mathcal{L}(X)$ be the set of all CDFs that arise as vague limits of sequences $\left\{F_{t_{k}}\right\}$ with $t_{k} \rightarrow \infty$ as $k \rightarrow \infty$. A sequence of CDFs is said to converge vaguely to a CDF $F$ if there exists a countable dense subset $D$ of $\mathbb{R}$ such that

$$
\lim _{n \rightarrow \infty}\left[F_{n}(b)-F_{n}(a)\right]=F(b)-F(a)
$$

for all $a, b \in D$. We use $\mathcal{L}$ to describe the asymptotic behavior of the stochastic process $X(t)$ as $t \rightarrow \infty$. The stochastic process $\{X(t), t \geq 0\}$ is said to be strongly stable if $F$ is proper for all $F \in \mathcal{L}$.

Theorem 4.1. The queue length process $X(t)$ in an $\mathrm{M}_{t} / \mathrm{M}_{t} / c(t)$ queue with general deterministic arrival and departure rates $\lambda(t)$ and $\mu(t)$, respectively, and a deterministic, time-varying number of servers $c(t)$ is strongly stable, and all CDFs in the limit set $\mathcal{L}$ have finite moments of all orders, if there exist positive numbers $t_{0}, \varepsilon$, and $T$ such that

$$
\int_{t_{0}+n T}^{t_{0}+(n+1) T} \lambda(v) \mathrm{d} \nu \leq(1-\varepsilon) \int_{t_{0}+n T}^{t_{0}+(n+1) T} c(v) \mu(\nu) \mathrm{d} v
$$

for $n=0,1,2, \ldots$

The proof is a straightforward generalization of the proof of Theorem 2.1 of [2] and, so, is omitted here. See also [1] for a related theorem about multiserver queues with periodic BMAP arrivals but a fixed number of servers.

We assume that (4.1) holds and that $\lambda(t), \mu(t)$, and $c(t)$ are periodic with period 1 , and define $X_{n}=X(s+n)$ for any fixed $s \in[0,1)$. Then, from Lemma 3.1 of [2], the embedded sequence $\left\{X_{n}\right\}$ is an irreducible, aperiodic, positive-recurrent Markov chain with stationary transition probabilities.

\section{Examples}

We consider a variety of simple examples with a maximum of two servers and constant service rate. In such cases, our equation for $p_{\hat{c}-1}(t)=p_{1}(t)$ becomes

$$
\begin{aligned}
p_{1}(t)=\int_{s}^{t} \mu p_{1}(\eta) & {\left[\max \{0, c(\eta)-1\} \tilde{I}_{0}(\eta, t)-c(\eta) \tilde{I}_{1}(\eta, t)\right.} \\
& \left.+\int_{\eta}^{t} \mathrm{e}^{-\Lambda(\eta, v)} \lambda(\nu) \tilde{I}_{0}(\nu, t) \mathrm{d} v\right] \mathrm{d} \eta+\tilde{I}_{0}(s, t)
\end{aligned}
$$

and our expression for $\mathrm{E}[X(t)]$, the expected number in the queue at time $t$, is

$$
\begin{aligned}
\mathrm{E}[X(t)]= & 1+\Lambda(s, t)-\mu \int_{s}^{t} c(v) \mathrm{d} v+\mu \int_{s}^{t} c(v) \int_{s}^{v} \mathrm{e}^{-\Lambda(\eta, v)} \mu p_{1}(\eta) \mathrm{d} \eta \mathrm{d} v \\
& +\mu \int_{s}^{t} \max \{c(v)-1,0\} p_{1}(v) \mathrm{d} \nu .
\end{aligned}
$$


TABLE 1: The piecewise-constant arrival $\left(\lambda_{1}, \lambda_{2}\right)$ and departure $(\mu)$ rates used in the examples.

\begin{tabular}{cccc}
\hline Example & $\lambda_{1}$ & $\lambda_{2}$ & $\mu$ \\
\hline 1(a) & 40 & 20 & 30 \\
$1(b)$ & 40 & 20 & 20 \\
2(a) & 30 & 30 & 30 \\
2(b) & 30 & 30 & 20 \\
$3(a)$ & 20 & 40 & 30 \\
$3(b)$ & 20 & 40 & 20 \\
\hline
\end{tabular}

We use these formulae to graph six examples with piecewise-constant rates and time-varying numbers of servers. In each of these examples, the number of servers drops from two to one halfway through the period and then returns to two at the beginning of the next period. Let $\lambda(t)=\lambda_{1}$ be the arrival rate for $t=n+s$, where $n$ is an integer and $s \in[0,0.5)$, and let $\lambda(t)=\lambda_{2}$ be the arrival rate for $t=n+s$, where $n$ is an integer and $s \in[0.5,1)$. In each example, $\mu$ is constant.

For each example given in Table 1 , we graph $p_{1}(t)$ and $\mathrm{E}[X(t)]$ over eight periods. These graphs are displayed in Figures 2-7. Example (a) in each case satisfies the stability condition given in Theorem 4.1, and tends to a periodic limit. In example (b) in each case, the average arrival rate equals the product of the service rate and the average number of servers. This does not satisfy the stability condition.
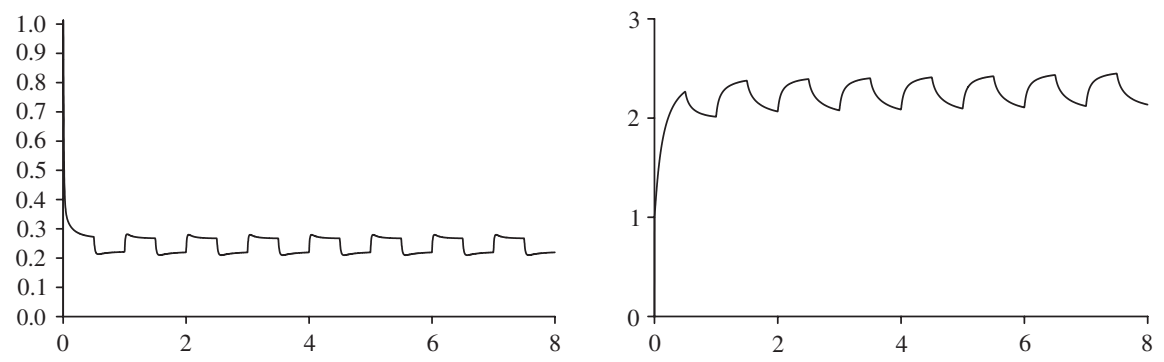

Figure 2: Example 1(a). Probability $p_{1}(t)$ (left) and expectation $\mathrm{E}[X(t)]$ (right) versus time (days) for $\lambda_{1}=40, \lambda_{2}=20$, and $\mu=30$.
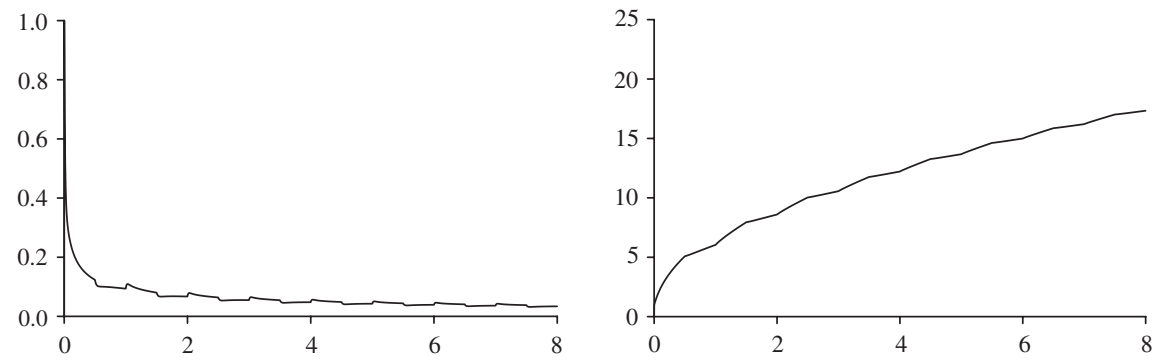

Figure 3: Example 1(b). Probability $p_{1}(t)$ (left) and expectation $\mathrm{E}[X(t)]$ (right) versus time (days) for $\lambda_{1}=40, \lambda_{2}=20$, and $\mu=20$. 

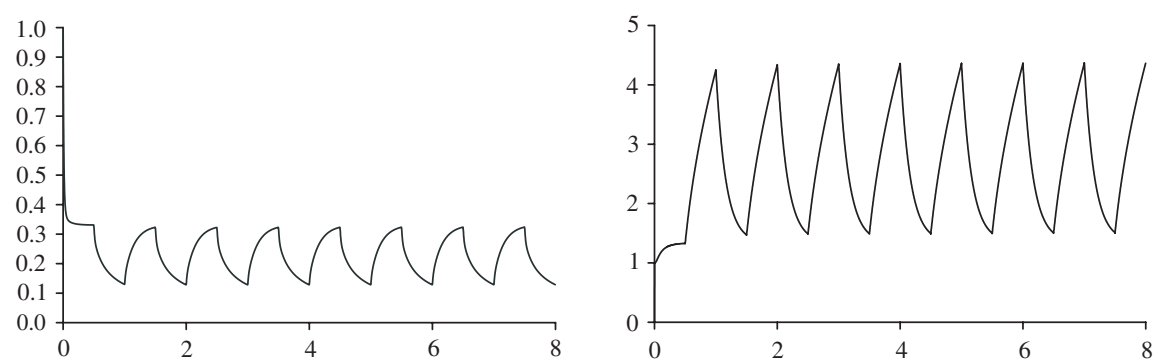

FIGURE 4: Example 2(a). Probability $p_{1}(t)$ (left) and expectation $\mathrm{E}[X(t)]$ (right) versus time (days) for $\lambda_{1}=30, \lambda_{2}=30$, and $\mu=30$.
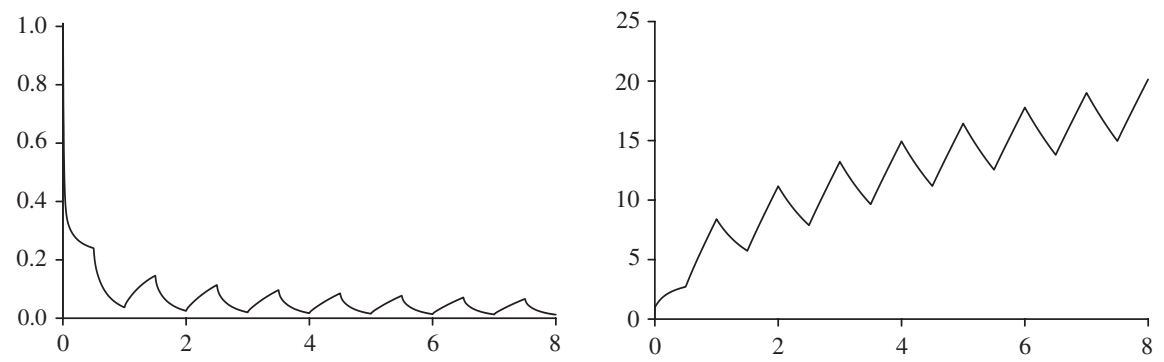

FIGURE 5: Example 2(b). Probability $p_{1}(t)$ (left) and expectation $\mathrm{E}[X(t)]$ (right) versus time (days) for $\lambda_{1}=30, \lambda_{2}=30$, and $\mu=20$.
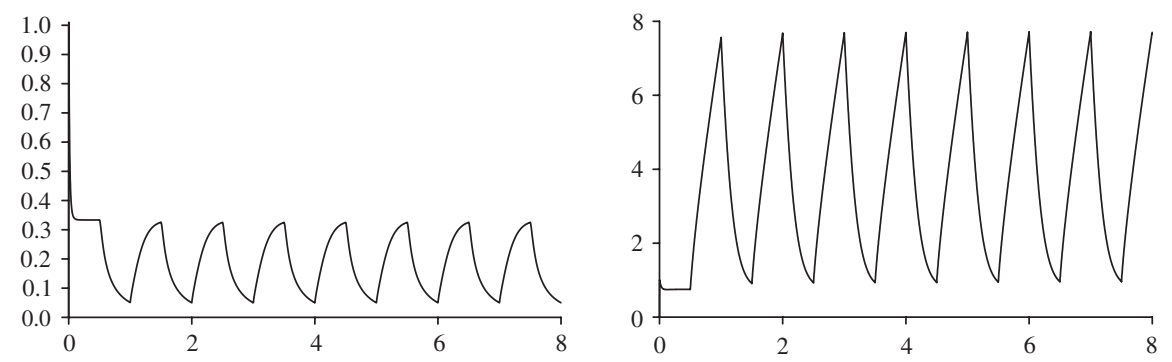

Figure 6: Example 3(a). Probability $p_{1}(t)$ (left) and expectation $\mathrm{E}[X(t)]$ (right) versus time (days) for $\lambda_{1}=20, \lambda_{2}=40$, and $\mu=30$.
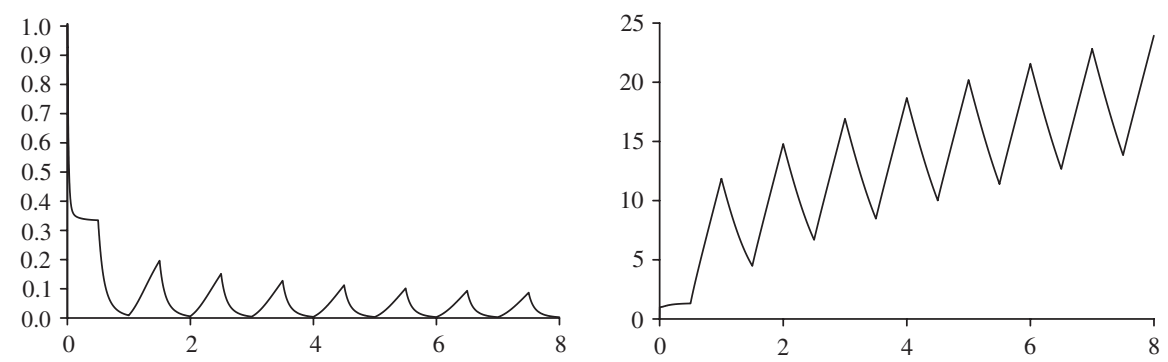

Figure 7: Example 3(b). Probability $p_{1}(t)$ (left) and expectation $\mathrm{E}[X(t)]$ (right) versus time (days) for $\lambda_{1}=20, \lambda_{2}=40$, and $\mu=20$. 


\section{Acknowledgements}

This paper is part of a cooperative effort between Cleveland State University and the City of Cleveland Division of Police. In particular, I want to thank Deputy Chief Prioleau Green of the Police Division, and John Holcomb and Teresa LaGrange at Cleveland State University. I am also appreciative of comments and improvements suggested by an anonymous referee.

\section{References}

[1] Breuer, L. (2001). The periodic BMAP/PH/c queue. Queueing Systems 38, 67-76.

[2] Heyman, D. P. and Whitt, W. (1984). The asymptotic behavior of queues with time-varying rates. J. Appl. Prob. 21, 143-156.

[3] Parthasarathy, P. R. and Sharafali, M. (1989). Transient solution to the many-server Poisson queue: a simple approach. J. Appl. Prob. 26, 584-594.

[4] Zhang, J. And Coyle, E. J. (1991). The transient solution of time-dependent M/M/1 queues. IEEE Trans. Inf. Theory 37, 1690-1696. 